ISSN: 0210-1696

DOI: https://doi.org/10.14201/scero202152e141161

\title{
EL PROCESO DE CONFINAMIENTO POR LA COVID-19 DEL ALUMNADO CON TRASTORNOS DEL ESPECTRO AUTISTA ESCOLARIZADO EN CENTROS ORDINARIOS: UN ANÁLISIS CUALITATIVO DEL IMPACTO SOBRE ELLOS, SUS NECESIDADES FUTURAS Y LAS DE SUS FAMILIAS ${ }^{1}$
}

The Process of Confinement of Learners with Autism Spectrum Disorders in Ordinary Schools due to COVID-19: A Qualitative Analysis of its Impact and Future Needs of Learners and their Families

Cecilia Simón Rueda

Universidad Autónoma de Madrid. Departamento Interfacultativo de Psicología Evolutiva y de la Educación

cecilia.simon@uam.es

Margarita Cañadas PÉREZ

Universidad Católica de Valencia. Centro de Atención Temprana "L'Alqueria”

M. a Luz FERnÁNDEZ BLÁZQUEZ

Universidad Autónoma de Madrid. Departamento Interfacultativo de Psicología Evolutiva $y$ de la Educación

Gerardo EcheITA SARRIONANDIA

Universidad Autónoma de Madrid. Departamento Interfacultativo de Psicología Evolutiva $y$ de la Educación

1 Este estudio se desarrolla en el marco del proyecto Análisis y valoración del proceso de educación inclusiva del alumnado con Trastornos del Espectro del Autismo desde educación infantil hasta la universidad: la participación social como eje de análisis (EITEA), financiado por el Programa Estatal de Investigación, Desarrollo e Innovación Orientada a los Retos de la Sociedad. Ministerio de Economía, Industria y Competitividad (EDU 2017-86739-R). 
Resumen: El estudio que se presenta tiene como objetivo principal "dar voz" a familias que tienen hijos con Trastornos del Espectro Autista para conocer el impacto que tiene en el proceso de enseñanza y aprendizaje de estos la situación de confinamiento generada por la COVID-19. Para ello, se ha contado con la participación voluntaria de 19 progenitores con hijos TEA escolarizados en centros ordinarios desde las etapas de educación infantil hasta la educación superior de dos comunidades autónomas. Como estrategia de recogida de información se han llevado a cabo cuatro grupos de discusión. Los resultados obtenidos muestran los apoyos prestados por la escuela a sus hijos y que han contribuido a su aprendizaje. Asimismo, han hecho hincapié en las dificultades encontradas que lo han limitado, la participación social de sus hijos como dimensión fundamental para las familias, el impacto de las demandas realizadas por parte del centro en el bienestar familiar, así como sus preocupaciones en relación con la incorporación de estos a la escuela. Como se mostrará, esta situación ha puesto de manifiesto factores importantes tanto de las políticas como de las prácticas de centro relevantes para los centros que se preocupan por la inclusión.

Palabras clave: educación inclusiva; alumnado con Trastornos del Espectro Autista; COVID-19; necesidades de las familias; necesidades del alumnado.

Aвstract: The main objective of the study is to "give voice" to families who have children with Autism Spectrum Disorders to understand the impact on the teaching and learning process that confinement has generated because of COVID-19. To this end, 19 parents who have children with ASD from two autonomous communities have been voluntarily participating. All of them are in ordinary schools from kindergarten to higher education. Four focus groups have been developed as an information collection strategy. The results obtained show the support provided to their children by the school and how this support has contributed to their learning. Furthermore, they underline the difficulties that have limited the learning process, and also the social participation of their children as a fundamental dimension for families. These results also show the impact of the demands made by schools on family well-being, as well as their concerns regarding the incorporation of their children into the school. As will be shown, this situation has highlighted important factors in both policies and practices that are important for schools concerned with inclusion.

KEYWORDs: inclusive education; learners with Autism Spectrum Disorders; inclusive education; COVID-19; family needs; learners needs.

\section{Introducción}

Quién IMAginaba A PRINCIPIOS DEL 2020 que se cerrarían todos los centros educativos y que permanecerían así hasta el final del curso?, ¿quién imaginaba, además, que los propios centros y las familias se encontrarían en una situación de gran incertidumbre respecto al comienzo del curso siguiente? Tampoco tenemos 
muy claro qué ocurrirá en un futuro, pero sí sabemos muy bien que esta situación ha impactado en todos los estudiantes y en sus familias, afectando muy directamente a su calidad de vida individual y familiar (Muñoz y Lluch, 2020).

Lo que también sabemos es que, una vez más, ese impacto ha sido desigual, ahondando en la injusta brecha entre quienes tenían y tienen buenas condiciones vitales y han podido, por ejemplo, "seguir aprendiendo en casa", gracias a los recursos tecnológicos y al apoyo familiar, y quienes no tenían ni lo uno ni lo otro y, por ello, no han podido hacerlo porque viven en situaciones de vulnerabilidad. Situaciones que se suman a otras ya previas como las que preocupaban entre otras muchas entidades a la Unesco (2018). Como señala el director global de Educación del Banco Mundial (Saavedra, 2020), se está generando una nueva brecha educativa cuyos impactos pueden ser diversos y graves para el desarrollo educativo de muchos alumnos y alumnas. En efecto, entre estas consecuencias se encuentran el abandono escolar estrechamente relacionado con las dificultades económicas que viven las familias; la continuidad de los estudios (riesgo mucho mayor en el alumnado más desfavorecido que suele tener menos oportunidades de aprendizaje fuera de la escuela); confusión y estrés en el profesorado; falta de preparación de las familias para el aprendizaje en casa y a distancia; a lo que se unen circunstancias personales, económicas y familiares de los propios padres (aspectos también relacionados con posibles lagunas en el cuidado de los niños y niñas o malnutrición en estos, y que nuevamente impactan más en las familias con menos recursos económicos y educativos); mayor exposición a la violencia y la explotación; dificultades para el seguimiento del aprendizaje del alumnado; incremento de las situaciones de riesgo a la violencia, y, además, el aislamiento social que supone la pérdida del contacto social, tan importante para su desarrollo y educación (Giannini y Grant Lewis, 2020) y para su regulación emocional (Ceballos y Sevilla, 2020). En este sentido, Unicef (2020) también llama la atención sobre el impacto de la crisis de la COVID-19 en la salud mental de la infancia.

Esta situación nos lleva ineludiblemente a preguntarnos ¿qué podemos aprender de lo ocurrido respecto al compromiso internacional de hacer efectivo el derecho a una educación de calidad con equidad?; ¿seguiremos como hasta ahora, donde ese derecho para algunos se da por sentado y para otros muchos es poco más que un desecho? (Echeita, 2017). A menos de diez años ya de llegar a la meta que fijaba la Agenda 2030 para la consecución de los Objetivos para el Desarrollo Sostenible en materia de educación (Objetivo n. ${ }^{\circ}$ 4): ‘ sabremos aprovechar esta oportunidad para repensar profundamente la educación escolar que tenemos, cargada de segregación, marginación y fracaso escolar?

Esta última pregunta es muy necesaria, especialmente al conocer los desalentadores análisis de la UNESCO (2020) respecto a los avances en este objetivo 4. Como se señala en ese informe, los mismos mecanismos excluyen y oprimen no solo a las personas con discapacidad, sino también a otras, a causa de factores como el género, la edad, el lugar donde se encuentran, la pobreza, la discapacidad, el origen étnico, la pertenencia a pueblos indígenas, la lengua, la religión, la migración o la situación de desplazamiento, la orientación sexual o la expresión de identidad de género, las creencias 
y actitudes. En definitiva, nos muestran cómo funciona la interseccionalidad que en su día estudiara la profesora Kimberlé Crenshaw (1989)².

Muchas preguntas que requieren un poco de sosiego y tiempo para pensar sobre ellas colectivamente, algo que, en parte, ofrecía esta anomalía planetaria que nos ha tocado vivir, y de la que también habrá algo bueno que deducir si nos damos la oportunidad de pararnos a pensar en lugar de salir precipitadamente hacia el mismo sistema educativo del que partíamos, excluyente e injusto para muchos.

Lo dicho no significa que este haya sido o esté siendo un tiempo perdido. ¡ $\mathrm{Ni} \mathrm{mu-}$ cho menos! Sin duda alguna podríamos reconocer logros muy importantes. Nosotros nos quedamos con los que conectan con lo más humano que tenemos: la disposición al cuidado y la solidaridad de tantas personas, organizaciones o empresas que se han volcado, precisamente, en apoyar a quienes más lo necesitaban, conforme a sus posibilidades y saberes.

El futuro que se presenta es incierto, pero también sabemos que no está escrito, sino que depende, entre otros muchos factores, de las respuestas a estos y otros interrogantes que nos interpelan como ciudadanos y también como educadores a quienes nos dedicamos a este empeño.

Uno de los retos en la educación, es dar respuesta a los estudiantes considerados con "necesidades educativas especiales", entre los que se encuentran los alumnos con Trastornos del Espectro Autista. Estos niños y jóvenes han tenido que adaptarse a tiempos inciertos, escuelas cerradas, cambio en las rutinas, la pérdida de conexiones con maestros y amigos y el miedo a contraer el virus, lo que, unido a otros posibles condicionantes familiares, ha generado mayor estrés, si cabe, para todas sus familias. Si bien la tecnología ha permitido la continuidad de la docencia, las personas con Trastornos del Espectro Autista pueden necesitar apoyo adicional para procesar las noticias y adaptarse a los muchos cambios. Estas poblaciones han tenido que enfrentar desafíos adicionales relacionados con la comprensión, la comunicación y la dificultad para relacionarse desde la distancia por vía online (Hume et al., 2020).

La familia es un nexo fundamental de unión entre escuela y el alumno o alumna con Trastornos del Espectro Autista y, en estos momentos más que nunca, se aprecia la importancia de los principios de las prácticas centradas en las familias (Cate et al., 2020), pues las familias están ayudando a estos niños y jóvenes a mantenerse en el proceso de aprendizaje escolar, a entender los cambios y a procesar sus sentimientos provocados por los mismos. Esta situación parece que está sirviendo para que profesorado y profesionales que no partían de este marco de prácticas centradas en la familia estén entablando ahora esa relación de colaboración con los cuidadores que tan esencial ha sido durante el confinamiento. Un marco de trabajo que, como bien sabemos, se aglutina alrededor de una participación activa de las familias para favorecer el desarrollo y aprendizaje de sus hijos e hijas, mediada por el asesoramiento profesional, con el fin de fortalecer sus

2 https://es.wikipedia.org/wiki/Kimberl\%C3\%A9_Crenshaw. 
competencias y ofrecer apoyos a partir de las prioridades y necesidades familiares (García Sánchez et al., 2014).

Precisamente, este trabajo pretende dar voz a familias que tienen hijos e hijas con Trastornos del Espectro Autista con el objetivo de: a) conocer el impacto de la situación de confinamiento generada por el COVID-19 en el alumnado con TEA en términos de aprendizaje y también de bienestar emocional desde la perspectiva de sus progenitores; b) las dificultades y los apoyos prestados por la escuela, y c) sus preocupaciones en relación con la incorporación a la escuela.

\section{Método}

\subsection{Participantes}

Se ha contado con la participación de 19 progenitores (7 padres y 12 madres) con hijos e hijas con TEA escolarizados en centros ordinarios de dos comunidades autónomas (Comunidad de Valencia y Comunidad de Madrid). Atendiendo a la etapa escolar de sus hijos e hijas, seis son progenitores de niños en Educación Infantil, en seis casos lo están en Educación Primaria, en tres en Educación Secundaria Obligatoria, en uno en Bachillerato, en uno en Formación Profesional y en dos su hijo está en la Universidad. Las familias se ubican principalmente en un nivel socioeconómico medio.

\subsection{Instrumentos}

Como estrategia de recogida de información se ha empleado el grupo de discusión. El grupo de discusión es una técnica que permite generar interacción y debate entre los participantes para expzlorar los puntos de encuentro y desencuentro con relación a las preguntas de investigación (Barbour, 2016). Siguiendo a la autora, el número recomendable de personas integrantes para esta técnica oscila entre los 3 y los 12 participantes. Así, se han llevado a cabo 4 grupos de discusión con una media de 5 participantes por grupo organizados por etapas educativas (Educación Infantil, Educación Primaria, Educación Secundaria y Bachillerato/Formación Profesional y Universidad). La duración de los grupos de discusión fue de 90 minutos aproximadamente.

Los grupos de discusión giraron en torno a cuatro preguntas centrales:

- ¿Cómo se ha desarrollado el proceso de confinamiento y la posterior desescalada?

- ¿Cómo ha impactado esta situación generada por el COVID-19 en tu hijo o hija, en términos de aprendizaje y también de bienestar emocional?

- ¿Qué apoyos habéis recibido por parte del centro que han sido útiles para vuestro hijo o hija y qué dificultades os habéis encontrado en la respuesta proporcionada por el centro?

- ¿Qué os preocupa de cara al futuro en relación con la incorporación a la escuela que se está proponiendo? ¿Qué le pediríais a los centros? 


\subsection{Procedimiento y análisis de datos}

Los participantes eran las familias que habían formado parte de un estudio previo realizado por el equipo de investigación en el marco del mismo proyecto de investigación, en su caso con la finalidad de construir relatos de vida de familias con hijos e hijas con TEA desde atención temprana hasta la educación superior. Se envió una carta a todas las familias participantes informando del estudio y solicitando su participación en el mismo con la información sobre su desarrollo.

Los grupos de discusión se realizaron en formato on line, a través de la plataforma Zoom y fueron grabados y transcritos con el correspondiente consentimiento por parte de las familias, proporcionando información del objetivo del estudio, asegurando el anonimato, la confidencialidad de la información, así como el uso de la misma con fines de investigación.

En los grupos de discusión se contaba con, al menos, tres miembros del equipo de investigación. Uno de ellos era el responsable principal de dinamizar la sesión y otro se responsabilizaba de anotar en un diario de campo (Jociles, 2015) aquellos sucesos relevantes de cara a los objetivos de investigación y el transcurso de la sesión. Estas transcripciones se sometieron al método de análisis temático propuesto por Braun y Clarke (2006).

En primer lugar, dos de los investigadores responsables del desarrollo de los grupos de discusión leyeron todas las transcripciones para familiarizarse con el contenido. Posteriormente generaron independientemente una propuesta de códigos que permitieran organizar la información reunida en las transcripciones. Las dos propuestas se compartieron y debatieron, lo que dio lugar a una lista enriquecida de códigos que supuso también la fusión de algunos. La codificación se llevó a cabo utilizando el programa informático NVivo v.12. A continuación los autores ordenaron los códigos en torno a temas y subtemas más amplios, que condensaban el significado de los códigos y reflejaban análisis más profundos (Saldaña, 2016). Los subtemas y temas se evaluaron en cuanto a homogeneidad interna y heterogeneidad externa (Corbin y Strauss, 2008). Por último, los autores reflexionaron conjuntamente sobre la contribución de cada uno de los temas y subtemas al conjunto de la información reunida, así como sobre la mejor manera de denominarlos con la finalidad de que reflejaran el contenido fundamental del tema tratado. Como garantías de la calidad del proceso de investigación, además de la realización del análisis conjunto, también se realizó triangulación entre investigadores (Cresswell, 2013; Flick, 2014) con el resto de miembros del equipo.

\section{Resultados}

Los temas generados, en torno a los que se han agrupado los códigos, son lo suficientemente amplios y genéricos como para que en ellos puedan tener cabida reflexiones y aportes procedentes de la diversidad de fuentes utilizada. Los temas en cuestión son los siguientes: 1 . Un contexto común de desconcierto y resignación; 2. Emociones 
desbordantes; 3. El confinamiento y su impacto sobre la vida familiar y las rutinas del alumnado; 4. Las pantallas y la educación on line; 5 . La actuación de los centros escolares; 6. El confinamiento y su impacto sobre la participación y el aprendizaje de los estudiantes; 7. La desescalada; 8. Empoderar y cuidar a los cuidadores.

Cada uno de los temas incluye en su título, entrecomilladas, expresiones literales de los participantes que buscan visibilizar el sentido del tema. Cada una de las citas incluidas en los resultados se acompaña de las siglas de la persona que la formuló y el rol que ocupa en la familia junto con la etapa educativa en la que está escolarizado su hijo.

\subsection{Un contexto de desconcierto y resignación. "Nadie estaba preparado para esto"}

A estas alturas es ya un lugar común señalar que la pandemia ha supuesto una situación para la que nadie estaba preparado y que ese desconcierto y todas sus preocupaciones y emociones asociadas nos han afectado a todas y todos, niños y adultos, en lo personal, lo social y, en su caso, en lo profesional. Los centros escolares, desde la Educación Infantil a la Universidad, son, en todas sus dimensiones (organizativas, curriculares, materiales, etc.), uno de esos contextos donde el impacto de la COVID-19 ha sido tremendo, obligando a buscar con urgencia máxima alternativas a la presencialidad, con acciones, tiempos, medios y normas - unas veces acertadas, otras no tantoplanteados con el fin de minimizar en lo posible el impacto negativo del confinamiento sobre la educación escolar de niños, adolescentes y jóvenes.

Hacer explícitas estas reflexiones, por otro lado bien conocidas por todos, tiene como finalidad reconocer que los análisis concernientes a cómo ha transcurrido esta anomalía educativa en el caso de los estudiantes con TEA y sus familias participantes en los grupos de discusión creados para este estudio no pueden hacerse al margen de este contexto general de desconcierto y preocupación, pues, como dice Slee (2020, p. 911), "para comprender la exclusión y perseguir la inclusión no podemos separar el objeto de análisis de su contexto”.

Apreciamos que ello ha tenido como consecuencia un cierto efecto mitigador de las críticas razonables vinculadas a situaciones desajustadas vividas por las familias, por ejemplo, en relación con la carga de trabajo que sus hijos recibían o a la falta manifiesta de coordinación del profesorado, en algunos casos.

Con la buena intención, pero quiero decir, que la misma persona que está intentando hacer ese trabajo (enseñar) teletrabajando también es madre, entonces dice "a ver, cómo lo hago para yo poder estar con mis hijos y al mismo tiempo dar la docencia a distancia y estar pendiente de mis hijos, del colegio, de la directora, del currículum, de cumplir los objetivos y, encima, no, es que 'no llego'", no te puedes organizar porque no has trabajado nunca ahí. También hay que ponerse ahí en ese lugar, que ha hecho cada uno lo que ha podido en la selva esta. (Padre de alumno en Educación Primaria)

Por otra parte, un elemento muy presente en ese contexto de gran y disperso impacto en todos (profesorado, familiares, estudiantes), y a la vez causa y producto de 
las circunstancias vividas, han sido las emociones de todo tipo, positivas y negativas, que han aflorado y han estado casi siempre presentes en las interacciones y dinámicas familiares, tanto en los momentos del trabajo escolar como en la vida cotidiana.

\subsection{Emociones desbordantes que todo lo tiñen. "Ha sido una experiencia superdura"}

Es evidente que las difíciles circunstancias generadas por la pandemia han tenido un impacto emocional de primer orden cuyas consecuencias a medio y largo plazo, en todos nosotros, están por determinar. Buena parte de esas emociones han sido de las que se consideran negativas: ansiedad, preocupación, miedo, frustración, rabia y han estado asociadas a los distintos impactos que, como veremos más adelante, pueden haber originado el confinamiento:

Ya es difícil nuestra vida, quieras o no. Era muy difícil antes de la cuarentena, y ahora sigue siéndolo. (Padre de alumna en Educación Infantil)

... ¿Tú ves mi cara, ves cómo estoy? A ver, que yo sé que una cosa es decir "esto, esto y esto", pero otra cosa es el día a día, y otra cosa es todo lo que cargamos los padres porque, acá, quieras o no los padres somos los que llevamos la peor parte o los que cargamos todo. Entonces, muchas veces es sí, otro día más de lucha, poco a poco, paso a paso, sí, sí, todo... Pero, vamos, los padres venimos quemados, yo me quemé. (Madre de alumna en Educación Infantil)

Pero también y, paradójicamente vista la dureza de la situación, han aflorado en algunos casos emociones y percepciones positivas:

El cambio de rutinas del colegio o del día a día, el encierro en sí, todo lo que ha significado para todos y lo duro que ha sido sobre todo para los adultos, creo yo, para los peques ha sido, vamos, lo han llevado fenomenal, y X en concreto nos ha sorprendido muchísimo porque no le hemos visto ningún cambio más allá de lo típico que decimos los padres cuando ya llevamos tres meses en plan "necesita colegio urgente", pero como, digamos, el desfogarse, el salir y demás, pero no como algo implícito del TEA en este caso. Entonces para nosotros la lectura del encierro ha sido positiva en cuanto a que no hemos visto demasiado problema. Es mi percepción. (Madre de alumno en Educación Infantil)

El grupo de amigos, digamos que tienen características como las de él, pues había comunicación por Skype u otra plataforma, prácticamente diaria, hasta que ya nos han abierto la puerta a poder salir, hacer ejercicio físico, cualquier otro tipo de cosas... Yo creo que él ha necesitado menos cosas que nosotros. (Madre de alumno en la Universidad)

Al mismo tiempo, los padres han podido, por una parte, apreciar y valorar el trabajo educativo que se hace en la escuela:

Y también uno se da cuenta de la importancia y de lo que se logra también en el cole, porque tú te das cuenta... Claro, ella iba de 9:30 hasta las $17 \mathrm{~h}$ y muchas veces uno dice, 
bueno, pero qué hará, qué le estarán enseñando, estarán enseñándole o no... Pero te das cuenta de lo importante que es también ese tiempo que está también en la escuela y todo lo que va recibiendo porque uno muchas veces, claro, uno la ve todos los días y muchas veces parece que no avanza, y uno quisiera que, claro, que esto fuera más rápido, que ella fuera aprendiendo más cosas, pero no es así y esto a nosotros como que nos dimos cuenta un poco más de su condición. El estar tanto tiempo con ella como nunca antes y tratar de... Los momentos ausentes también de ella, entonces es muy difícil. (Padre de alumna en Educación Infantil)

Pero también han podido apreciar directamente, a cuenta de estar con sus hijos continuamente y de ver a sus compañeros a través de la pantalla, las diferencias que en términos de dificultades puede tener su hijo o hija y el progreso que se deriva de una acción educativa que necesariamente debe tener su ritmo:

Y, también, uno tiene la posibilidad que no tiene todos los días de ver a sus demás compañeritos, entonces, claro, también uno ve la diferencia entre niños de la misma edad que ella y cómo ya se desenvuelven, entonces, eso también, quieras o no es... Fue duro. (Padre de alumna en Educación Infantil)

\subsection{El confinamiento y su impacto sobre la vida familiar y las rutinas del alumnado. "Sobreviviendo, sobreviviendo..."}

El teletrabajo de los padres, no siempre en condiciones idóneas, ni mucho menos, junto con la atención a los hijos y el seguimiento de la clases on line de estos, ha sido un contexto propicio para que las rutinas de las familias y las de los propios hijos se hayan visto afectadas, sea en detalles aparentemente sin importancia ("ahora no consiente en calzarse”) o sea en aspectos más preocupantes como propiciar un cierto "abuso de las pantallas" como estrategia para que, durante algunos ratos, los padres puedan dedicarse a sus propias obligaciones. Rutinas que, si bien es cierto que para todos los niños tienen una gran importancia, mucho más para aquellos con TEA, y tanto para el desarrollo psicomotor como para la atención u otros procesos cognitivos:

Entonces en casa ha sido muy difícil trabajar con él... ahí sí que nos sentíamos nosotros mal porque es que con $\mathrm{X}$ prácticamente no hemos trabajado nada porque ha sido un caos el teletrabajo; los dos teletrabajando, los niños en casa, la mayor que acababa de pasar a $1 .^{\circ}$ de primaria, mil tareas, mil aplicaciones para subir por aquí, por allá... Y ha sido un completo caos y el pobre $\mathrm{X}$ se ha quedado como en $2 .^{\circ}$ plano, con esa tranquilidad que nosotros teníamos un poco de que iba superbién, pero sí que es verdad que lo hemos dejado un poquito a la suya y de ahí creo yo la comodidad esta que tiene él ahora de "no quiero volver al cole", en casa estoy superbien, veo la tele, veo los dibujos, que antes estaban más restringidos o cojo la tablet y hago un poquito mi libre albedrío. Entonces eso ha sido el caos del COVID en casa, supongo que como en todas las casas con niños, con teletrabajo y demás. (Madre de alumno en Educación Infantil) 
Conciliar la vida laboral, familiar y escolar no ha sido una tarea nada fácil para las familias -en ocasiones casi una misión imposible-, las cuales han tenido que echar mano de todos los recursos a su alcance, empezando por las pantallas. Estas son una ayuda que actúa cual arma de doble filo, pues pueden ayudar tanto como desajustar la influencia educativa de otras acciones o estímulos.

En nuestro caso las pantallas son un problema porque absorben mucho y dificultan la interacción social. Nosotros necesitamos que él salga, hable, juegue y todo el mundo, todos los niños normales, por decirlo de alguna manera, se han volcado con las pantallas; los padres estaban como nosotros, trabajando a tope. (Padre de alumno en Educación Secundaria)

Por otra parte, que todos los niños y las niñas desde muy temprana edad estén familiarizados con su uso no es garantía absoluta de que la formación que se apoya en ellas sea eficaz. Ello dependerá de otros muchos factores.

Los padres, conscientes de ello, lo han vivido en muchas ocasiones de forma muy estresante:

Estábamos trabajando los dos 8 horas, de 8 de la mañana a 17:30, y el niño estaba completamente solo, o sea, me refiero que estábamos los dos en casa, pero él estaba en el salón jugando con tablet o con vídeos del YouTube, $\mathrm{X}$ y yo cada dos por tres teniendo que ir corriendo a echarle un a ver, cada vez que escuchábamos un ruido, cada vez que no lo escuchábamos porque decíamos "por dios, qué está haciendo". Y, entonces, claro, fue una situación superagobiante en ese aspecto porque, por eso, es que requiere tantísima atención y no le estás dando ninguna atención a tu hijo en ese aspecto. (Madre de alumno en Educación Infantil)

\subsection{Las pantallas y la educación on line. "A mí me cuesta competir con el móvil"}

Para los hijos e hijas de las familias participantes en este estudio, el acceso a las pantallas -como expresión para referirse al uso de juegos, aplicaciones o acceso a vídeos o programas de TV y, en su caso, a información sobre sus intereses, a través de móviles, tabletas u ordenadores-, se ha convertido en un compañero inseparable de sus vidas y una fuente inagotable de entretenimiento. La diferencia, en algunos casos, con sus iguales sin TEA puede ser su tendencia a un uso excesivo que, a la larga, puede ser muy perjudicial.

(Pasados los primeros 15 días de confinamiento) nosotros cada vez necesitábamos más recursos para que no se aburra y, entonces, cada vez más móvil, que eso después nos dimos cuenta de que se nos fue de las manos porque le dábamos el móvil para que ella se entretenga y ya llegó un momento que también se nos fue de las manos, eso lo reconocimos porque después era móvil, móvil, televisión, YouTube... (Madre de alumna de Educación Infantil) 
... Yo implicada con ella y que estaba acostumbrada a trabajar con ella de cierta forma, cuando el móvil fue cada vez ganando espacio a mí me cuesta competir con el móvil y con las propuestas del móvil y con todo, con los colores, con la música... Y, claro, yo ahora le pongo algo y no, no, se pasa y está muy adicta a pantallas, a la tablet, al televisor, a todo lo que sea eso. Y, bueno, en parte eso es lo que más nos crea una sensación de agobio, frustración. (Madre de alumna en Educación Infantil)

Pero como todo instrumento, su bondad o perjuicio no está en sí mismo, sino en su uso y, en este sentido, como veíamos más arriba, es posible que el confinamiento haya propiciado un abuso de su utilización. Pero también es cierto que, en el caso de algunos estudiantes más mayores, ese dominio de las pantallas les ha facilitado, en cierto sentido, la tranquilidad que sus padres necesitaban y el aprovechamiento de las clases on line.

Bueno, $\mathrm{X}$ tiene problemas especiales para manejar el ordenador, tiene una cierta habilidad para ello y entonces enseguida pues se adaptaba a cómo funcionaban las distintas plataformas que se le ofrecían y, bueno, ha sido más una cuestión de gestionar más el tiempo, de establecer una rutina: me tengo que levantar a tal hora, tengo el examen en tal fecha, tengo que planificar la agenda. (Padre alumno en la Universidad)

Además, era un medio idóneo para minimizar las relaciones sociales cara a cara que, como es bien sabido, son costosas para el alumnado con Trastornos del Espectro Autista:

En nuestro caso no nos ha pedido el salir, él con las nuevas tecnologías está aquí a gustísimo y no ha querido nunca salir, de hecho, durante el confinamiento no salimos y, nada, lo ha llevado bastante bien, pero eso, con las nuevas tecnologías... (Padre de alumno en Educación Primaria)

Claro, el tema, cuando hay esta distancia, cuando es on line la participación, AJ es una máquina porque apenas hay interacción social, con lo cual él en ese sentido no ha tenido ningún problema, no ha tenido ningún desajuste social que es lo que tiene, más o menos, X, su comportamiento en clase... Bueno, él tiene ya unas pautas determinadas, pero, bueno, que impensablemente se producen desajustes sociales, y eso no lo ha vivido porque era online. (Madre de alumno en Bachillerato)

Pero este dominio y gusto por las pantallas por parte de la mayoría del alumnado con TEA no debe interpretarse como un facilitador per se para la formación on line. Más bien parece que en algunos casos, al menos con los más pequeños, esta se ha ajustado muy poco a sus competencias comunicativas y sociales y ha podido ser un medio, incluso, para que afloren relaciones sociales negativas:

Él no quería conectarse nunca, era como nombrarle el colegio y yo no sé, de ir tan a gusto, eso no sé por qué, la verdad es que no lo llego a entender muy bien, de ir súper a gusto, supercontento, genial, no ha llorado más allá de los 15 primeros días del cambio de cole, vamos, lo normal, como cualquier otro niño de la clase durante el periodo de adaptación. Jugar con todos los niños, la profesora comentarnos que en 
cuanto a relaciones es uno más en la clase, a de repente, yo no sé si es que él el verlo aquí con los cuadraditos todos los niños, unos llorando, otros chillando, les pasaba a más, he de decir, había otros nenes de su clase que tampoco se querían conectar, también les pillaba la hora de la siesta... No sé, pero es verdad que X no ha querido: las dos veces que se ha conectado, ha sido al brazo mío, se giraba, no quería mirar a cámara, no quería decir nada. En cambio, luego, sí que me ha pedido llamar por videollamada a algunos amiguitos de forma particular y sí que han estado hablando nada, dos minutos y se aburren y se van, pero sí que lo ha pedido; pero con la profesora y con la clase no quería. (Madre de alumno en Educación Infantil)

\subsection{La actuación de los centros escolares. "Buena voluntad, mucho desajuste"}

Como señalábamos anteriormente, se han apreciado pocas críticas crudas a la labor de los centros escolares y sus equipos docentes a lo largo de las distintas etapas educativas. Las familias han sido muy conscientes de lo inesperado de este proceso y por ello de que nadie estaba preparado para lo que tenía que venir. Ello no quita para que cuando tienen ocasión de reflexionar sobre lo sucedido aprecien muchos elementos de mejora en muy variados aspectos. Otra cosa distinta es que ahora ellos y nosotros juzguemos si se podría haber iniciado mucho antes una formación para que el profesorado estuviera mejor capacitado para la enseñanza on line que se venía venir desde hace muchos años (Fernández Enguita, 2018), junto con una preparación de los medios necesarios para ello (espacios, dispositivos, capacidad de la red, etc.).

Lo que yo veo en general, hablo de todos los colegios, lo que hace falta es que ningún colegio en esta situación está preparado para el tema de trabajar a distancia, ni por protocolos, ni por medios, absolutamente de ninguna forma, y lo que ha ocurrido es que se han aturullado también a decir "vamos a ver cómo cumplimos". (Padre alumno en Educación Primaria)

En todo caso, si hay una crítica importante a la acción docente, aunque quede en cierta medida edulcorada por el atenuante de las circunstancias, esa es el desajuste de la carga de trabajo y de los deberes que los docentes enviaban y la capacidad tanto de los padres para asumirla (en tanto que mediadores ahora del proceso de enseñanza), así como de sus hijos o hijas para asimilarla a tenor de sus capacidades y conocimientos previos. Se trata de un desajuste que ha quedado también de manifiesto en el estudio e informe elaborado por la Confederación Española de Asociaciones de Padres de Alumnos (CEAPA)

Las familias percibían una tendencia por parte del profesorado a proponer una sobrecarga de tarea a sus hijos e hijas:

A ver, con respecto a la valoración, estamos encantadísimos con el colegio por el afecto de seguimiento, ha sido un seguimiento totalmente diario. Lo que nosotros todos los días,

3 CEAPA. "Las familias piden que dosifiquen las excesivas tareas a distancia”. https://www.publico. es/sociedad/familias-piden-profesores-dosificar-excesivas-tareas-distancia.htm.l 
lo que pasaba es que recibíamos un correo con todas las actividades que teníamos que hacer con X. Entonces, ¿qué es lo que pasaba? Que muchas veces yo decía, esta muchacha, dios mío, no es capaz de hacer eso que me está pidiendo en esta actividad porque es que yo sé los límites que tiene mi hijo, entonces digo: “¿Esta tía qué se ha fumado?”. Es imposible, entonces básicamente le decía: "Esto no lo voy a hacer” y empezaba: "Sí, pero es que tienes que hacer porque lo tienes que intentar". Y digo: "Sí, sí, que lo que tú quieras”, pero que, si tengo tiempo, que vale, pero yo no me puedo poner a hacer una actividad de imprime, recorta, plastifica, vuelve a recortar, vuelve a poner no sé qué para poder hacer una actividad en concreto. Lo haré, de hecho, lo he hecho varias veces que me juntaba a lo mejor con 20-30 actividades, me dedicaba un día a recortar, a colorear, plastificar... Y le dejaba todo el material preparado para el día siguiente o lo que sea pues para ponerme a trabajar cosas que llevaba atrasadas de semanas anteriores. (Madre de alumno en Educación Infantil)

Como era de esperar y no queriendo las familias que sus hijos o hijas quedaran más descolgados, algunos padres y madres no han tenido reparo en reconocer que han sido ellos quienes han realizado algunas de esas tareas ante la imposibilidad de que sus hijos las finalizaran por sí mismos a tiempo:

El tema era a la hora de ponerte con el niño a hacer los deberes, es que era mucha más tarea que lo habitual, entonces tenías que hacerla sí o sí, y muchas cosas las hemos presentado los padres. Yo le hacía a mi hijo los trabajos de plástica porque era incapaz de ponerse conmigo a hacer los trabajos de plástica, y se lo dije a los profesores, que sepáis que todo lo que ha presentado él lo hemos hecho su padre y yo. (Madre de alumno en Formación Profesional)

A este notable desajuste en la carga de trabajo, percibido y sentido por las familias, se ha de sumar, sobre todo en el caso de los más mayores, el hecho de una falta de coordinación entre docentes a estos efectos, descoordinación agravada en ocasiones por la existencia de distintas plataformas o medios y por la diferente frecuencia de comunicación entre cada uno con las familias de sus respectivos grupos. También han echado de menos una atención más continuada hacia las necesidades específicas de apoyo educativo de sus hijos o hijas:

Él necesitaba a su lado alguien que le dijera: "Esto está bien hecho, esto no está bien hecho”. Entonces yo sí que he echado en falta, por ejemplo, la presencia de alguna persona de apoyo más continuamente porque sí le llamaban una o dos veces en semana, pero el resto del tiempo no. (Madre de alumno en Formación Profesional)

Pero yo el principal problema que he visto es que la transmisión no estaba preparada, entonces cada uno utilizó una plataforma distinta, no había ningún criterio. A nosotros nos llegaban por 4-5 sitios diferentes lo que pedían los profesores. Entonces tenías que hacer una labor semanal de ponerte al día de lo que había para la semana siguiente, luego planificarlo, revisar diariamente, darte cuenta de que había gente que mandaba 16 correos electrónicos y si no te los leías te perdías parte de los deberes; otros tíos que no escribían nunca; gente a la que no le llegaba; gente que no sabía manejar la plataforma 
como profesor, entonces... Entonces ha sido un sindiós, pero porque ellos mismos no estaban preparados y la propia dirección del centro tampoco era capaz de implantar una doctrina, ... (Padre de alumno en Educación Secundaria)

\subsection{El confinamiento y su impacto sobre la participación y el aprendizaje de los estudiantes. "¿Y sus relaciones sociales qué?”}

Llegados a este punto es imprescindible volver a señalar la intrínseca diversidad de todo el alumnado, a la cual no son ajenos los alumnos y alumnas con TEA. Esa diversidad se refleja, entre otros muchos aspectos, en sus competencias cognitivas, lingüísticas o sociales, que, por otra parte, van variando significativamente con la edad. Por todo ello es imposible e imprudente hacer generalizaciones con relación al impacto del confinamiento en el aprendizaje y participación del alumnado con TEA. Lo que sí cabe es poner de manifiesto casuísticas que tienen la capacidad de contradecir estereotipos y prejuicios que algunas personas pueden tener; por ejemplo, que este alumnado no puede aprender "ni llegar lejos" o que "no les gustan las relaciones sociales y prefieren estar solos".

En el caso de $\mathrm{X}$ yo creo que necesita ver a los profesores cuando le explican porque él necesita ver su expresión para comprenderles, pero el contacto social le agota, le cansa mucho porque tiene que hacer un esfuerzo que otras personas no tienen que hacer. Entonces, pues ha sacado unas notas muy buenas porque realmente su esfuerzo ha estado centrado al estudio y como ha seguido en contacto con sus amigos, ha estado jugando con ellos a través de internet, pues, bueno, el tema social pues no lo ha visto reducido ni se ha visto afectado. (Madre de alumno en Educación Secundaria)

Pero cuando hablamos de alumnado con TEA, las preocupaciones por las relaciones sociales están al orden del día. De hecho, para algunas familias se han convertido en una de sus fuentes principales de preocupación.

Él a nivel de tecnología se maneja perfectamente, hace años que tiene ordenador, iPad y todo, y lo que tuvimos que hacer fue estructurar un trabajo para que él tuviera una disciplina porque sabíamos que era muy necesario para él no dejar que se abandonara a quedarse en su cuarto porque nosotros la principal preocupación que tenemos es conseguir la integración social. (Padre de alumno en Educación Secundaria)

Ahora no, hay niños que se le acercan y, claro, ella como no habla y no puede... Es como que se queda ahí, se queda en blanco, y esa es la parte que más me preocupa o que veo mayor impacto, en la sociabilidad, le hace falta estar con otros niños. (Madre de alumna en Educación Infantil)

Pues como X prácticamente, lo que más hemos echado en falta es todo el tema social, ha sido brutal porque es que él estaba superacostumbrado a socializar con más compañeros de clase, con más niños, y es que ha pasado de un día para otro a estar solo con su padre y conmigo, sin nadie más. Entonces pues ahora es verdad que él en el parque pues ni se acerca a niños ni nada... El tema social y el tema de rutinas fatal porque es que le 
hemos sacado... Él estaba seguro en su entorno del colegio, tenía sus horarios, él iba a su cole, luego iba a sus terapias $\mathrm{y}$, de pronto, se ha quedado sin terapia, sin cole, sin nada... (Madre de alumno en Educación Infantil)

Lo paradójico del confinamiento es que, si bien la limitación de relaciones sociales ha sido una preocupación constante de muchas familias -en particular con los niños más pequeños-, en algunos casos esta misma limitación ha sido un cierto alivio para algunos de ellos:

Como ha seguido en contacto con sus amigos, ha estado jugando con ellos a través de internet, pues, bueno, el tema social pues no lo ha visto reducido ni se ha visto afectado. (Madre de alumno en Educación Secundaria)

$\mathrm{La}$ ansiedad social esta que tiene también él le ha venido muy bien el bajar el nivel de contactos porque $\mathrm{X}$ va a diferentes deportes... para que tenga núcleos sociales diferentes, (para que) tenga que relacionarse y, bueno, le gusta mucho el deporte. Y quizás, quitarle todo eso a él le ha favorecido un poco porque eso le genera a él mucha ansiedad. (Madre de alumno en Formación Profesional)

Si el proceso de confinamiento fue duro para todos, la desescalada, que parecía, de entrada, un alivio seguro a las preocupaciones de las familias y una posibilidad para la mejora del bienestar emocional de los estudiantes con TEA, no siempre ha sido así.

El problema más gordo que está teniendo X con todo el confinamiento es que ya no consiente ponerse zapatos y va todo el día andando de puntillas, eso ha ido para atrás, pero, vamos... Incluso la terapeuta del centro de Atención Temprana que se encarga de eso, también nos ha llamado, nos ha dado truquitos, nos ha dado cosas que podemos trabajar con él para eso, pero... Pero, vamos, que no, ahí sí que no... Igual que el tema de estimulación sí que está mucho más avanzado, pero el tema de fisioterapia, por así decirlo, ahí ha ido para atrás, pero bestial. (Madre de alumno en Educación Infantil)

\subsection{La desescalada. "No precisamente fácil"}

Paradójicamente, el confinamiento, que había creado una burbuja de seguridad y una intensa relación entre padres e hijos, también ha sido, en muchas ocasiones, una barrera para recuperar su nivel de relaciones sociales con otros y sus oportunidades para explorar el entorno.

Él lo que ha llevado muy mal ha sido la desescalada, yo creo que habíamos creado como un núcleo que estábamos él y yo solos y ha sido... Yo empecé a trabajar fuera, cuando pasamos a la fase 1... y ahí lo empezó a pasar muy mal, fue a partir de ahí. Más la desescalada que el confinamiento porque lo asumió y estábamos aquí en una burbujita y estaba bien. Pero, claro, a mí me ha dado pena porque él lo que necesita aprender es todos los hábitos, todas las interacciones... Había creado muchísimo vínculo con los compañeritos, hicimos alguna vez videoconferencia y en ese sentido me ha dado mucha pena porque eran cosas que necesitaba, bueno, y ha sido salir del confinamiento... Bueno, y después pasamos una época que no había manera de salir de casa, que tenía miedo y no quería salir de casa. Cuando por fin pudimos empezar a salir de casa y se ha ido relajando pues ahora 
busca muchísimo más, lo noto yo, estar con otros niños que es una cosa positiva. Pero que lo duro ha sido la desescalada para nosotros. (Madre de alumno en Educación Primaria)

Y en la desescalada igual, con mucho cuidado porque él es verdad que todo esto de la suciedad como que lo lleva un poco mal... X es, bueno, me imagino que, como la mayoría de los autistas, es muy obsesivo, entonces se pone la mascarilla y tiene que tener la mascarilla súper... Además, tiene gafas, entonces superbién colocada, se pasa tres años poniéndose la mascarilla y luego para que no salga... En fin, cuando nos preparamos para ir a la calle a él le tengo que avisar como media hora antes: "Vete poniéndote la mascarilla”. (Madre de alumno en Bachillerato)

Como es sabido, después de la desescalada no hubo vuelta al cole, sino que las familias que pudieron empalmaron con las vacaciones o el descanso de los meses de verano a la espera de un curso nuevo lleno de expectativas y preocupaciones.

Si se cumple el peor de los pronósticos y dicen que empezamos el curso igual que lo hemos terminado, pues hombre, si fuera la misma profesora y tal, pues todavía, pero si lo cambian, claro... Si ya le va a costar con todos los niños, pues como le venga alguna como esta desafiante un poquillo que le intente tomar la medida a través de la pantalla, pues, claro, es que va a ser complicado. Y, bueno, a nivel académico pues llegaremos hasta donde podamos, yo ya tampoco me voy a obsesionar porque no me da, se hará lo que se pueda y demás. $\mathrm{Y}$ con respecto a lo que decía $\mathrm{X}$, tienes toda la razón, es que nosotros somos padres y ahora hemos asumido un rol o hemos tenido que asumir un poco el rol de profesores en casa y yo con $\mathrm{X}$, no sé vosotros, pero yo con $\mathrm{X}$ ha sido complicado porque poco menos que me decía: “Tú eres mi madre, no eres mi profesora”. (Madre de alumno en Educación Primaria)

No, pero nosotros tenemos una doble preocupación: por un lado, que cambia de centro y la adaptación a un nuevo centro igual que a un niño normal, entre comillas, pues un niño con diversidad funcional no sabemos hasta dónde le va a afectar, no le va a afectar. Y luego el tema de la aplicación de las medidas de seguridad, cómo pueden ellos aplicar, X que está siempre chupándose el dedo... Es muy difícil. (Padre de alumno en Educación Infantil)

Dificultades, ansiedad y dudas que han puesto a prueba la resiliencia de las madres y padres de estos niños y adolescentes, y con ello la necesidad de que también deben cuidarse si quieren, como quieren, seguir cuidando a sus hijos y promoviendo su desarrollo y felicidad.

\subsection{Empoderar y cuidar a los cuidadores. "Yo tengo que estar bien para poder ayudar a mi bija, eso es básico"}

La realización de estos grupos de discusión no ha sido valiosa solamente para reconocer lo que las familias demandan, esperan y ciertamente se necesita para dar una respuesta inclusiva de calidad a las necesidades educativas de sus hijos o hijas en el contexto de esta pandemia que todavía no ha pasado. También han sido una oportunidad para tomar conciencia de que como madres y padres también tienen necesidades propias de muy distinto tipo, al margen de sus hijos, que deben reconocer y tratar de satisfacer pues, de lo contrario, ponen en riesgo sostener los cuidados y la atención 
que ellos y ellas necesitan, así como su capacidad de luchar por el cumplimiento cabal de sus derechos. Y obviamente también arriesgan su propio equilibrio y bienestar emocional al que tienen igual derecho.

$\mathrm{Al}$ preguntar a las familias qué le pedirían al centro estas piden, sobre todo, comprensión de la situación familiar y de sus necesidades como forma de apoyo al bienestar familiar, en la medida que toman conciencia de que este es importante para poder responder adecuadamente a las necesidades de sus hijos.

Un poco de contención, no sé si psicológica o un poco de ese entendimiento, comprensión a los padres que venimos agotados, quemados con todo esto, y de un poco de ver esa parte de cómo estás, cómo te sientes, te parece que puedes esto... No es mucho... O no sé, esa parte de contención a los padres sobre todo primero porque estoy segura de que yo tengo que estar bien para poder ayudar a mi hija, eso es básico. Si yo no estoy bien o me siento mal o estoy... Yo no puedo ayudar a mi hija, Yo no puedo ayudar a mi hija, que es lo que me pasó algunos días, no puedo ayudarla porque yo misma estoy cansada, estoy agotada, estoy quemada... Entonces no me sale nada, mi paciencia es diferente con mi hija, entonces esa parte, un poco más de atención a los padres. (Madre de alumna en Educación Infantil)

Y también algo de seguimiento o apoyo más continuo en las tareas a desarrollar. Como señala la familia de X (Madre de alumno en Educación Secundaria)

Tanto mi marido como yo hemos echado en falta, es que $\mathrm{X}$, yo no sé si les pasa a los vuestros, pero $\mathrm{X}$ necesita una persona siempre a su lado que le vaya diciendo un poco el camino que tiene que tomar, entonces, él necesitaba a su lado alguien que le dijera "esto está bien hecho, esto no está bien hecho". Entonces yo sí que he echado en falta, por ejemplo, la presencia de alguna persona de apoyo más continuamente porque sí le llamaban una o dos veces en semana, pero el resto del tiempo no. Entonces sí que he necesitado esa persona ahí a su lado que es el que le entiende, que está con él en el cole continuamente y ahora no ha estado. Eso es lo que hemos echado en falta nosotros. (Madre de alumno en Educación Secundaria)

Con todo y con ello y si bien es cierto que esta no ha sido sensu stricto una investigación inclusiva (Parrilla y Susinos, 2013), sí ha servido para contribuir, aunque sea modestamente, al empoderamiento de las familias que tan generosamente estuvieron dispuestas a participar.

Queremos agradecerles a ustedes por tenernos en cuenta para este debate que, quieras o no, nos hace bien, a nosotros, pues nos hace bien contar nuestra experiencia y saber que no estamos tampoco solos en esto y que no somos los únicos. Cada uno, todo tiene su particularidad, pero, bueno, nos hace bien poder ayudar a quien sea que esté pasando por eso. Gracias por tenernos en cuenta. (Madre de alumno en Educación Infantil)

\section{Conclusiones}

El trabajo realizado en este estudio nos ha permitido conocer de primera mano experiencias específicas vividas por las madres y los padres participantes en el mismo durante el periodo de confinamiento originado por la pandemia de la COVID-19, junto 
con sus hijos e hijas con TEA y el resto de miembros de sus respectivas familias. Hemos tenido ocasión de profundizar en cómo ha transcurrido el proceso de confinamiento derivado de la COVID-19 y los primeros pasos tras la llamada desescalada en dirección a la llamada "nueva normalidad", en casos que van desde niños o niñas que recién iniciaban su escolarización en la etapa de 3 a 6 años hasta jóvenes que realizaban el Bachillerato o recién habían ingresado en la Universidad. Se trataba en todos los casos, como se ha apuntado, de procesos vividos en el marco de su escolarización en centros ordinarios.

Si bien es cierto que nadie negaría la enorme diversidad de situaciones, experiencias y necesidades educativas y de otro tipo derivadas de las circunstancias singulares y propias de cada estudiante, de cada familia y de cada centro educativo en el que estaban escolarizados, creemos que es importante señalar que, dentro de esta diversidad, tenemos como denominador común un "déficit de realidad"; por ejemplo, se han cancelado los procesos de socialización e interacción física (Iglesias et al., 2020). Cuando llegó la pandemia y mandó a parar... la enseñanza presencial en los centros educativos lo que realmente se ha producido es una "enseñanza remota de emergencia” (Bozkurt y Sharma, 2020).

Desde esta situación de enseñanza a distancia/confinamiento común, la diversidad se ha visto reflejada, por ejemplo, en el manejo de la situación de estrés derivada del confinamiento por parte de las familias o respecto a las reacciones del alumnado con TEA a las directrices y tareas escolares que los centros remitían a las familias y su predisposición para la comunicación on line. Por su parte, para las madres y padres, que han tenido que añadir precipitadamente a sus habituales funciones parentales la de profesor o profesora, no es lo mismo bregar con las tareas manipulativas y de baja dificultad de los niños en Educación Infantil, que con algunos contenidos de Secundaria o Bachillerato. Y es obvio que sus circunstancias laborales, la propia vivienda y sus recursos son tan distintos, y en ocasiones desiguales, que resulta tremendamente simplificador considerarlos únicamente bajo el común denominador de padres o madres que tienen hijos o hijas con TEA.

Con todo y con ello es importante resaltar que hablamos de familias que se ubican en un nivel socioeconómico medio, lo que hace que su realidad y recursos para el afrontamiento de la calamidad que ha supuesto el confinamiento quede muy lejos de otras familias en contextos de extrema o gran vulnerabilidad.

Como esas "disclosing tablets" de las que nos hablara Sapon-Shevin (2013), la pandemia ha servido para sacar a la luz muchas de las injustas desigualdades se en las que estaba asentado el sistema educativo español y, obviamente, también en otros países. Algunas de estas desigualdades se han tratado de paliar desde las diferentes normativas, instrucciones y orientaciones que han publicado las administraciones españolas, tanto a nivel nacional como autonómico, durante el periodo de confinamiento, difiriendo entre ellas, pero con cierto consenso en torno a algunas cuestiones pedagógicas como "no dejar a nadie atrás" (Díez-Gutiérrez y Gajarzo, 2020). A pesar de estos esfuerzos, la "brecha digital" ha estado presente (Giannini y Grant Lewis, 2020), ha supuesto una desigual distribución tanto respecto al acceso como a un uso educativo de los recursos tecnológicos y redes necesarios para una educación en red de calidad, en la que las familias puedan participar. Esto, como señalan Muñoz y 
Lluch (2020), va mucho más allá del uso precipitado de los recursos disponibles que se ha hecho en estos meses pasados durante el confinamiento:

La suspensión de clases obliga a plantear otras opciones para que las familias atiendan educativamente a sus hijos e hijas en casa. Confiar todo a las lecciones online, las plataformas educativas y recursos digitales y Apps o los grupos de alumnos por WhatsApp, puede resultar un tanto peligroso. (Muñoz y Lluch, 2020, p. 7)

Otra de las debilidades que han salido claramente a la luz ha sido la relacionada con la cultura de colaboración existente en los centros escolares. Allí donde se ha puesto de manifiesto que dicha colaboración no estaba bien instaurada o era débil, la consecuencia ha sido una respuesta desajustada frente a las necesidades educativas específicas de determinados alumnos, alejando la urgente tarea de promover un aprendizaje personalizado que, no solo para el alumnado con TEA, sino también para la mayoría, ha quedado en buena medida arrasado por la pandemia y las condiciones escolares en las que esta ha acontecido.

Finalmente, no podemos dejar de reflexionar sobre un efecto muy dañino de la COVID-19 sobre la educación escolar de todo el alumnado y que este estudio también ha puesto de manifiesto. Este no ha sido, a nuestro juicio, la pérdida de clases para cubrir el temario, como se suele decir, sino las restricciones que el confinamiento ha puesto a la participación social tal y como nosotros la estamos analizando en nuestro proyecto (EDITEA, 2020). Sin oportunidades para reforzar el sentido de pertenencia (Prince y Hadwin, (2013), como ha ocurrido con el confinamiento, no solo acontece que el aprendizaje se ve afectado, sino que el desarrollo psicosocial de todos se verá muy afectado, siendo que los más vulnerables verán multiplicada esta desventaja.

Es por ello que el sentido de pertenencia, su ausencia y su deseo, se ha revelado en este periodo de confinamiento como primordial. En efecto, por eso era frecuente ver en las redes en esos días cómo las escuelas mandaban vídeos de su claustro unido o mandaban fotografías de su alumnado tratando, en definitiva, de tirar de ese sentido de pertenencia porque, sin la menor duda, es una necesidad básica del ser humano (Maslow, 1978).

Siempre se ha dicho que lo peor de la adversidad es que pueda dejarte doblemente herido; en el momento en el que la sufres y porque te imposibilite un futuro sin esperanza. Esa esperanza, como en su día explicara Fullan (2003, p. 125), "no es una visión ingenua y luminosa de la vida. Es la capacidad de no entrar en pánico en situaciones graves y de encontrar modos y recursos para abordar problemas difíciles". El mejor modo de abordar esos problemas difíciles es la disposición a trabajar juntos, unidos por un fuerte sentimiento de pertenencia, colaboración y apoyo mutuo. Si la crisis de la COVID-19 nos fuerza y nos lleva "realmente" a repensar el sistema educativo desde estos parámetros, en el marco de una nueva ecología de la equidad (Ainscow et al., 2013), entonces las preocupaciones y quebrantos de estos tiempos, que muchas familias han querido y necesitado compartir, no habrán sido en balde. El futuro no está escrito; de nosotros dependerá si las próximas crisis sanitarias "que pueden llegar" nos encuentran educativa y socialmente preparados o nuevamente distraídos con lo superficial. 
EL PROCESO DE CONFINAMIENTO POR LA COVID-I9 DEL ALUMNADO CON TRASTORNOS

DEL ESPECTRO AUTISTA ESCOLARIZADO EN CENTROS ORDINARIOS

C. SIMÓN, M. CAÑADAS, M. ${ }^{2}$ L. FERNÁNDEZ Y G. ECHEITA

\section{Referencias bibliográficas}

Ainscow, M., Goldrick, S. y West, M. (2013). Promoviendo la equidad en educación. Revista de Investigación en Educación, 11(3), 44-56.

BArbour, R. (2016). Los grupos de discusión en investigación cualitativa. Morata.

Bozkurt, A. y Sharma, R. C. (2020). Emergency remote teaching in a time of global crisis due to CoronaVirus pandemic. Asian Journal of Distance Education, 15(1), 1-5. https:// doi:10.5281/zenodo.3778083

Braun, V. y Clarke, V. (2006). Using thematic analysis in psychology. Qualitative Research in Psychology, 3(2), 77-101. https://doi:10.1191/1478088706qp063oa

Cate, D., Dell, P., Littlefield, R., Riepe, B. y Whaley, K. (2020, 23 de septiembre). Reaffirming key early childhood practices during a pandemic. Ecta Center. https://ectacenter. org/topics/disaster/coronavirus-key.asp

Ceballos, N. y Sevilla, S. (2020). El efecto del aislamiento social por el Covid-19 en la conciencia emocional y en la comprensión lectora. Estudio sobre la incidencia en alumnos con trastornos de aprendizaje y menor acceso a las Nuevas Tecnologías. Revista Internacional de Educación para la Justicia Social, 9(3), 1-13.

Corbin, J. y Strauss, A. (2008). Basics of qualitative research. 3. ${ }^{a}$ ed. Sage.

Cresswell, J. W. (2013). Qualitative inquiry and research design: choosing among five traditions. SAGE.

Díez Gutiérrez, E. J. y Gajardo Espinoza, K. (2020). Políticas educativas en tiempos de coronavirus: la confrontación ideológica en España. Revista Internacional de Educación para la Justicia Social, 9(3), 83-101. https://doi:10.15366/riejs2020.9.3.005

Echeita, G. (2017). Educación inclusiva. Sonrisas y lágrimas. Aula Abierta, 46, 17-24.

EDITEA. (2020, 9 de abril). Repensando la participación educativa en el contexto de una pandemia sanitaria. Diario de la Educación. https:/eldiariodelaeducacion.com/2020/04/09/ repensando-la-participacion-educativa-en-el-contexto-de-una-pandemia-sanitaria/

Fernández Enguita, M. (2018). Más escuela y menos aula: la innovación en la perspectiva de un cambio de época. Morata.

FLICK, U. (2014). La gestión de la calidad en la investigación cualitativa. SAGE.

Fullan, M. (2003). Sobre el cambio educativo. En A. Hargreaves (Ed.), Replantear el cambio educativo. Un enfoque innovador. Amorrortu.

Iglesias, E., González-Patiño, J., Lalueza, J. L. y Esteban-Guitart, M. (2020). Manifiesto en tiempos de pandemia: por una educación crítica, intergeneracional, sostenible y comunitaria. Revista Internacional de Educación para la Justicia Social, 9(3), 181-198. https:// doi:10.15366/riejs2020.9.3.010

Jociles, M. I. (2015). La observación participante en el estudio etnográfico de las prácticas sociales: pautas para observarlas/describirlas. En D. LoRENTE (Coord.), Metodología y trabajo de campo etnográfico (pp. 1-32). Instituto Nacional de Antropología e Historia.

García Sánchez, F. A., Escorcia, C., Sánchez López, C., Orcajada, N. y Hernández, N. (2014). Atención Temprana centrada en la familia. Siglo Cero, 45(3), 6-27.

Giannini, S. y Grant Lewis, S. (2020, 25 de marzo de). Three ways to plan for equity during the coronavirus school closures. GEM Report. https://gemreportunesco.wordpress. $\mathrm{com} / 2020 / 03 / 25 /$ three-ways-to-plan-for-equity-during-the-coronavirus-school-closures/

Hume, K., Waters, V., Sam, A., Steinbrenner, J., Perkins, Y., Dees, B., Tomaszewski, B., Rentschler, L., Szendrey, S., McIntyre, N., White, M., Nowell, S. y Odom, S. (2020). Supporting individuals with autism through uncertain times. Chapel Hill, NC: School of

Ediciones Universidad de Salamanca / CC BY-NC-ND

Siglo Cero, vol. 52, número extraordinario, 2021, pp. 141-161 
Education and Frank Porter Graham Child Development Institute, University of North Carolina at Chapel Hill. Recuperado de: https://afirm.fpg.unc.edu/sites/afirm.fpg.unc. edu/files/covid-resources/Supporting\%20Adults\%20with\%20Autism\%20through\% 20 Uncertian\%20Times\%20Full\%20Packet_0.pdf

Maslow, A. (1987). Motivation and personality (3. ${ }^{a}$ ed.). Harper and Row.

Muñoz Moreno, J. L. y Lluch Molins, L. (2020). Educación y Covid-19: colaboración de las familias y tareas escolares. Revista Internacional de Educación para la Justicia Social, 9(3). Recuperado de https://revistas.uam.es/riejs/article/view/12182

Parrilla, A. y Susinos, T. (2013). Investigación inclusiva en tiempos difíciles. Certezas provisionales y debates pendientes. REICE, 11(2), 88-98.

Prince, E. J. y Hadwin, J. (2013). The role of a sense of school belonging in understanding the effectiveness of inclusion of children with special educational needs. International Journal of Inclusive Education, 17(3), 238-262. https://doi:10.1080/13603116.2012.676081

SaAvedra, J. (2020, 20 de marzo). COVID-19 y educación: algunos desafíos y oportunidades. Education for Global Development. https://blogs.worldbank.org/es/education/educational-challenges-and-opportunities-covid-19-pandemic

SALDAÑA, J. (2016). The coding manual for qualitative researchers ( $3 .^{\text {a }}$ ed.). SAGE.

SAPOn-Shevin, M. (2013). La inclusión real. Una perspectiva de justicia social. Revista de Investigación Educativa, 11(3), 71-85.

SLEE, R. (2019). Belonging in an age of exclusion. International Journal of Inclusive Education, 23(9), 909-922. https://doi:10.1080/13603116.2019.1602366

Unesco. (2018). Reunión Mundial sobre la Educación 2018. Declaración de Bruselas. Recuperado de https://unesdoc.unesco.org/ark:/48223/pf0000366394?posInSet=1\&queryId=f00b beb5-caf0-495d-9782-e4caad1e9e0f

Unesco. (2020). Global Education Monitoring Report Summary. Inclusion and Education: All Means All. Unesco. Recuperado de: https://es.unesco.org/gem-report/report/2020/ inclusion

Unicef. (2020). Salud Mental e infancia en el escenario de la COVID-19: propuestas de Unicef España. Dirección de Sensibilización y Políticas de Infancia. Recuperado de https://www. unicef.es/sites/unicef.es/files/comunicacion/COVID19_UNICEF_Salud_Mental.pdf 


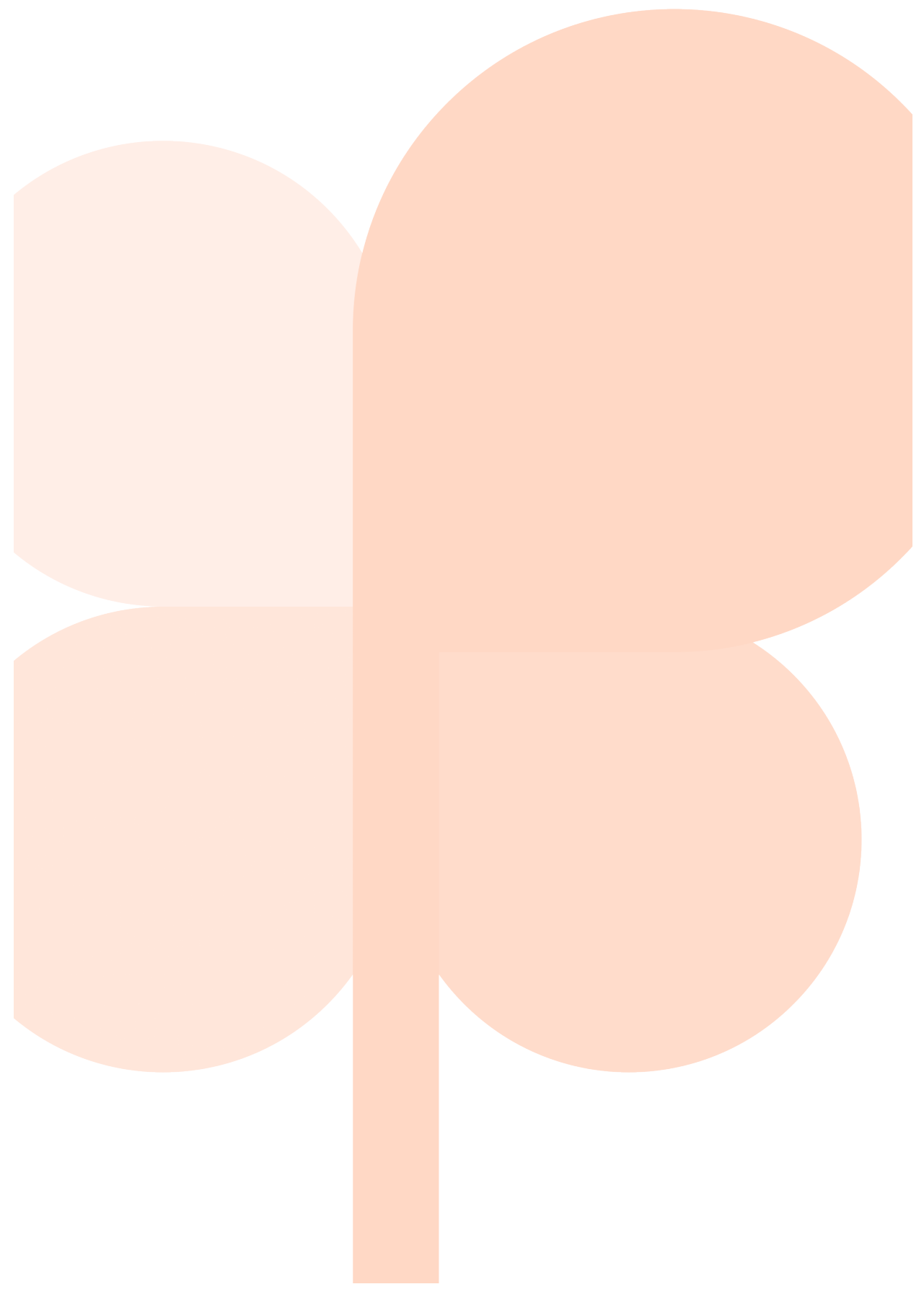

